\title{
Performance comparison of power delay profile Estimation for MIMO OFDM
}

\author{
Manisha K. Ahirrao, Beena R. Ballal ,Shraddha Panbude \\ Electronics and Telecommunication Department Vidyalankar Institute Of Technology \\ Wadala, Dadar (w), Mumbai, India
}

\begin{abstract}
A multiple-input multiple-output (MIMO) communication system combined with the orthogonal frequency division multiplexing (OFDM) modulation technique can achieve reliable high data rate transmission over broadband wireless channels. In linear minimum mean square error channel (MSE) estimation for multicarrier system, it is necessary to know channel correlation function. Hence estimation of noise variance using null carrier and power delay profile can be used to approximation which can be described in two parameters like; mean delay and root mean square (RMS) delay spread. The approximate power delay profile is used to generate LMMSE coefficient for subcarrier channel estimation. In this paper the estimation of channel at pilot frequencies with Minimum Mean Square (MMSE) estimation algorithms is carried out through Mat lab simulation. The performance of MIMO OFDM is evaluated on the basis of Mean Square Error (MSE) level.Simulation results shows the performance of MSE for estimated PDP and Constant PDP for varying number of samples and for SNR. The performance of LMMSE channel estimation using the proposed PDP estimate approaches to that of Wiener filtering while in the other case, the estimated PDP gives less SNR than constant PDP.
\end{abstract}

Keywords: - Channel estimation, Power delay profile, Mean Square Error, MIMO, OFDM.

\section{INTRODUCTION}

Orthogonal frequency division multiplexing (OFDM) is a multi-carrier transmission technology in wireless environment, and can also be seen as a multi-carrier digital modulation or multi-carrier digital multiplexing technology [9]. The basic idea of OFDM is to divide available bandwidth into $\mathrm{N}$ narrow subchannel at equidistant frequencies.[8]. A large number of orthogonal sub-carriers are used to transmit information. OFDM system has high utilization of frequency spectrum and satisfactory capability of reducing multi-path inference. So, OFDM has been considered as one of the core technologies of 4 th generation $(4 \mathrm{G})$ wireless communication system in the future Orthogonal Frequency Division Multiplexing. [1]

A single stream of data is split into parallel streams each of which is coded and modulated on to a subcarrier, a term commonly used in OFDM systems. OFDM is a multicarrier system uses discrete Fourier Transform/Fast Fourier Transform (DFT/FFT), $\sin (\mathrm{x}) / \mathrm{x}$ spectra for subcarriers .Available bandwidth is divided into very many narrow bands, Data is transmitted in parallel on these bands. In OFDM each subcarrier has a different frequency, Frequencies chosen so that an integral number of cycles in a symbol period, Signals are mathematically orthogonal and Data is carried by varying the phase or amplitude of each subcarrier. Orthogonal frequency division multiplexing (OFDM) transmission scheme is another type of a multichannel system, which employs multiple subcarriers, it does not use individual band limited filters and oscillators for each sub channel and furthermore, the spectra of subcarriers are overlapped for bandwidth efficiency, The multiple orthogonal subcarrier signals, which are overlapped in spectrum, can be produced by generalizing the single-carrier In practice, discrete Fourier transform (DFT) and inverse DFT (IDFT) processes are useful for implementing these orthogonal signals. DFT and IDFT can be implemented efficiently by using fast Fourier transform (FFT) and inverse fast Fourier transform (IFFT), respectively.

Single carrier system Signal representing each bit uses the entire available spectrum. In multicarrier system available spectrum divided into many narrow bands data is divided into parallel data streams each transmitted on a separate band

\section{PILOT BASED OFDM MODEL}




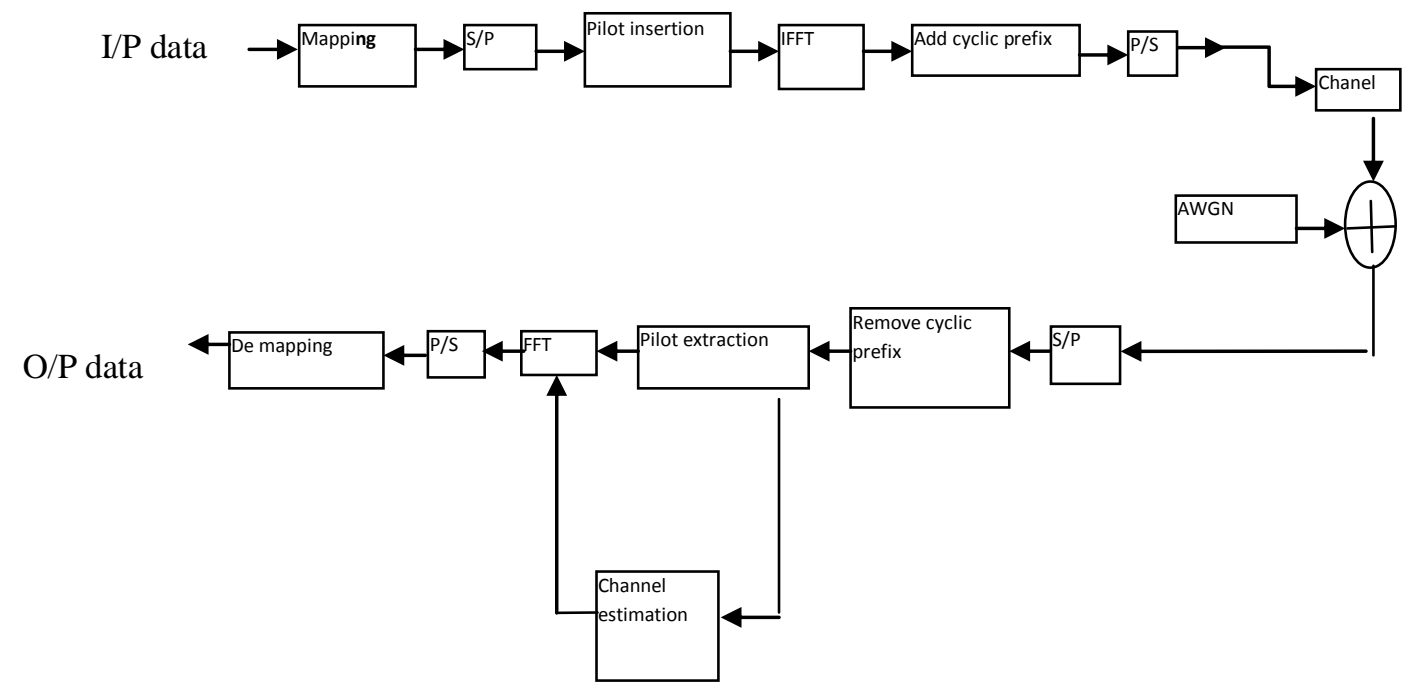

Fig 1: Pilot based OFDM system model.

Channel estimation plays a very important role in OFDM system .Many related algorithms have been presented these years, which can be generally separated into two methods, pilot-based channel estimation and blind channel estimation. In paper [4] the present channel estimation methods generally can be divided into two kinds: One is based on blind channel estimation which does not use pilots. Other is based on the pilots

\section{Blind channel estimation}

pilot-based channel estimation is a practical and an effective method[1]. Blind channel estimation, this uses statistical information of the received signals. Blind channel estimation methods avoid the use of pilots and have higher spectral efficiency. However, they often suffer from high computation complexity and low convergence speed since they often need a large amount of receiving data to obtain some statistical information such as cyclo stationarity induced by the cyclic prefix. Therefore, blind channel estimation methods are not suitable for applications with fast varying fading channels. And most practical communication systems such as World Interoperability for Microwave Access (WIMAX) system adopt pilot assisted channel estimation.

\section{Pilot channel estimation}

Pilot-based channel estimation estimates the channel information by obtaining the impulse response from all sub carriers by pilot. [1]

Pilot based channel estimation is based on the transmission of symbols that are known to receiver so called pilot symbols[4]. The pilot symbols are inserted into data stream and transmitted over mobile channel, at receiver pilot symbols are analyzed in order to obtain channel estimate which is utilized for equalization .As the characteristic of mobile channel is varying with time and frequency in order to obtain estimate which provides information about time and frequency domain channel variation, pilot symbol need to be transmitted periodically in time and spread over whole bandwidth which is provided for data transmission. For the pilotaided channel estimation methods, there are two classical pilot patterns, which are the block-type pattern and the comb-type pattern. The block-type refers to that the pilots are inserted into all the subcarriers of one OFDM symbol with a certain period i.e. symbols are transmitted periodically, and all subcarriers are used as pilots.[2] The block-type can be adopted in slow fading channel, that is, the channel is stationary within a certain period of OFDM symbols. The comb-type refers to that the pilots are inserted at some specific subcarriers in each OFDM symbol. The comb-type is preferable in fast varying fading channels [7], that is, the channel varies over two adjacent OFDM symbols but remains stationary within one OFDM symbol. The comb-type pilot arrangementbased channel estimation has been shown as more applicable since it can track fast varying fading channels, compared with the block-type one. 


\section{SYSTEM MODEL}

The system under consideration is a MIMO-OFDM system with $P$ transmits and $Q$ receive antennas, and $K$ total subcarriers [3].Suppose that the MIMO-OFDM system with the specified antennas transmits $K_{d}$ subcarriers at the central spectrum assigned for data and pilots, in order to control interferences with other systems. Let $\left[k_{p}\right.$, $n_{p}$ ] be the pilot subcarrier for the $p$ th transmit antenna at the $n_{p}$ th OFDM symbol, which is a QPSK modulated signal

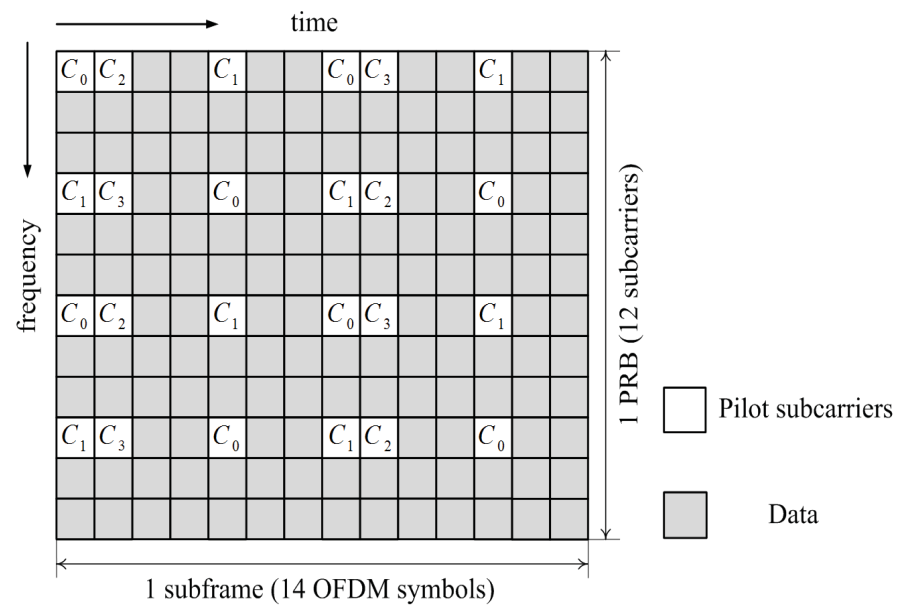

Fig : 2 Pilot symbol arrangement in a block of OFDM system [3]

We assume that the pilot subcarriers are distributed over a time and frequency grid as in Fig. 2, to preserve the orthogonality of pilots among different transmits antennas. At the $n_{p}$ th OFDM symbol, the number of pilot subcarriers is defined as $K p=p$. The pilot inserted OFDM symbol is transmitted over the wireless channel after performing an inverse fast Fourier transform (IFFT) and adding a CP. It is assumed that the length of CP, $L_{g}$, is longer than the channel maximum delay, $L_{c h}$, making the channel matrix circulate $\left(L_{c h} \leq L_{g}\right)$. At the receiver, after perfect synchronization, the removal of CP, and FFT operation, the received pilot symbol for the $q$ th receive antenna can be represented as, $\mathrm{y}_{\mathrm{q}}\left[\mathrm{n}_{\mathrm{p}}\right]=\operatorname{diag}\left(\mathrm{X}_{\mathrm{p}}\right) \mathrm{F}_{\mathrm{p}} \mathrm{h}_{\mathrm{p}, \mathrm{q}}+\mathrm{n}_{\mathrm{q}}$

where $\mathrm{h}_{\mathrm{p}, \mathrm{q}}=\left[\mathrm{h}_{\mathrm{p}, \mathrm{q}}\left[\mathrm{n}_{\mathrm{p}}, 0\right], \mathrm{h}_{\mathrm{p}, \mathrm{q}}\left[\mathrm{n}_{\mathrm{p}}, 1\right], \ldots \ldots ., \mathrm{h}_{\mathrm{p}, \mathrm{q}}\left[\mathrm{n}_{\mathrm{p}} \mathrm{L}_{\mathrm{ch}}\right], 0, \ldots ., 0\right]^{\mathrm{T}}$ is an $L_{g} \times 1$ CIR vector at the $p$ th transmit antenna and $q$ th receive antenna. [3]

\section{PROPOSED METHOD FOR PDP ESTIMATION:}

The proposed method is as follows:

From (1), the CIR at the $(p, q)$ th antenna port can be estimated approximately using the regularized least squares (RLS) channel estimation with a fixed length of $L_{g}$ as $\hat{\mathrm{h}}_{\mathrm{R}, \mathrm{p}, \mathrm{q}}=\left(\mathrm{F}_{\mathrm{p}}^{\mathrm{H}} \mathrm{F}_{\mathrm{p}}+\in \mathrm{I}_{\mathrm{Lg}}\right)^{-1} \mathrm{~F}_{\mathrm{p}}^{\mathrm{H}} \operatorname{diag}\left(\mathrm{X}_{\mathrm{p}}\right)^{\mathrm{H}} \mathrm{y}_{\mathrm{q}}\left[\mathrm{n}_{\mathrm{p}}\right]$

$$
\triangleq \mathrm{W}_{\mathrm{RLS}, \mathrm{p}} \mathrm{y}_{\mathrm{q}}\left[\mathrm{n}_{\mathrm{p}}\right]
$$

Where $\epsilon=0.001$ is a small regularization parameter, and $\mathbf{I}_{L g}$ is the $L g \times L g$ identity matrix. To derive the PDP from the estimated CIR in (2), the ensemble average of $\hat{h}_{\mathrm{R},}, \hat{\mathrm{h}} \mathrm{H}$ is given by

$\mathrm{E}\left\{\hat{\mathrm{h}}_{\mathrm{R}, \mathrm{p}, \mathrm{q}} \hat{\mathrm{h}}_{\mathrm{R}, \mathrm{p}, \mathrm{q}}^{\mathrm{H}}\right\}=\mathrm{WR}_{\mathrm{hh}} \mathrm{W}^{\mathrm{H}}+\sigma_{\mathrm{n}}^{2} \mathrm{~W}_{\mathrm{RLS}, \mathrm{p}} \mathrm{W}_{\mathrm{RLS}, \mathrm{p}}^{\mathrm{H}}$

Where $\mathrm{R}_{\mathrm{hh}}=\mathrm{E}\left\{\mathrm{h}_{\mathrm{p}, \mathrm{q}} \mathrm{h}_{\mathrm{p}, \mathrm{q}}^{\mathrm{H}}\right\}$ and $\mathrm{W}=\left(\mathrm{F}_{\mathrm{p}}^{\mathrm{H}} \mathrm{F}_{\mathrm{p}}+\in \mathrm{I}_{\mathrm{L}, \mathrm{g}}\right)^{-1} \mathrm{~F}_{\mathrm{p}}^{\mathrm{H}} \mathrm{F}_{\mathrm{p}}$

$\mathbf{R}_{h h}$, represent the PDP of multipath channel within the length of $L_{g}$, Unfortunately, $\mathbf{R}_{h h}$ is distorted by $\mathbf{W}$, which is an ill-conditioned matrix due to the presence of $\mathbf{F}_{H} \mathbf{F}_{p}$. Thus, instead of calculating $\mathbf{W}-1$, we investigate the method for eliminating the spectral leakage of $\mathbf{W}$. The covariance matrix of the estimated CIR is defined as $\mathbf{R}_{\widehat{h} \widehat{h}}=\mathbf{W R}_{h h} \mathbf{W}_{H}$ which can be expressed as

$\mathrm{R}_{\widehat{h} \hat{h}}=\sum_{l=0}^{\mathrm{L}_{\mathrm{g}}-1} \mathrm{~W} \operatorname{diag}\left(\mathrm{p}_{\mathrm{l}} \mathrm{u}_{\mathrm{l}}\right) \mathrm{W}^{\mathrm{H}}$

Where $\mathrm{u}_{l}$ is a unit vector with the $l$ th entry being one and otherwise zeros. Let $\mathrm{P}_{\widehat{h}}$ and $\mathbf{t}_{l}$ be the $L_{g} \times 1$ vectors defined as $\mathrm{P}_{\widehat{\mathrm{h}}}=\left(\widehat{\mathrm{R}}_{\widehat{\mathrm{h}}}\right)$ and $\mathbf{t}_{l}=D_{g}\left(\mathbf{W} \operatorname{diag}\left(\mathbf{u}_{l}\right) \mathbf{W} H\right)$, respectively, where $D g(\mathbf{A})$ is the column vector containing all the diagonal elements of $\mathbf{A}$. Then, the relation in (4) is simplified as

$\mathrm{P}_{\widehat{\mathrm{h}}}=\mathrm{p}_{0} \mathrm{t}_{0}+\mathrm{p}_{1} \mathrm{t}_{1}+\cdots .+\mathrm{p}_{\mathrm{Lg}-1} \mathrm{t}_{\mathrm{Lg}-1} \triangleq \mathrm{TP}_{\mathrm{h}}$

where $\mathbf{T}=[\mathbf{t} 0, \mathbf{t} 1, \ldots, \mathbf{t} L g-1]$ is defined as a distortion matrix by $\mathbf{W}$.. In addition, the distortion matrix is a wellconditioned matrix. Hence, the distortion of $\mathbf{W}$ can be eliminated as

$\mathrm{p}_{\mathrm{h}}=\mathrm{T}^{-1} \mathrm{P}_{\widehat{\mathrm{h}}}=\mathrm{E}\left\{\mathrm{g}_{\mathrm{p}, \mathrm{q}}\left[\mathrm{n}_{\mathrm{p}}\right]\right\}-\sigma_{\mathrm{n}}^{2} \widetilde{\mathrm{w}}$

where $\left.\mathrm{g}_{\mathrm{p}, \mathrm{q}}\left[\mathrm{n}_{\mathrm{p}}\right]=\mathbf{T}-1 D g(\hat{\mathrm{h}} R, p, q) \hat{\mathrm{h}} H R, p, q\right)$ is defined as the received sample vector for estimating PDP at the ( $p$, $q)$ th antenna port on the $n p$ th OFDM symbol, and $\widetilde{\mathrm{w}}=\mathbf{T}-1\left(\mathbf{W} R L S, p \mathrm{~W}_{\mathrm{RLS}, \mathrm{p}}^{\mathrm{H}}\right)$. 


\section{PDP Estimation in MIMO-OFDM Systems}

The received sample vector in (6) can be expressed as

$\mathrm{g}_{\mathrm{p}, \mathrm{q}}\left[\mathrm{n}_{\mathrm{p}}\right]=D g\left(\mathrm{~h}_{\mathrm{p}, \mathrm{q}} \mathrm{h}_{\mathrm{p}, \mathrm{q}}^{\mathrm{H}}\right)+\tilde{\mathrm{n}}_{\mathrm{p}, \mathrm{q}}+\mathrm{e}_{\mathrm{p}, \mathrm{q}}$

where $\tilde{\mathrm{n}}_{\mathrm{p}, \mathrm{q}}=\mathrm{T}^{-1} D_{g}\left(\mathbf{W}_{R L S, p} \mathbf{n}_{q} \mathrm{n}_{\mathrm{q}}^{\mathrm{H}} \mathrm{W}_{\mathrm{RLS}, \mathrm{p}}^{\mathrm{H}}\right)$ and

$\mathrm{e}_{\mathrm{p}, \mathrm{q}} \quad=2 R_{\mathrm{e}}\left\{\mathrm{T}^{-1}\left(\mathrm{Wh}_{\mathrm{p}, \mathrm{q}} \mathrm{q}_{\mathrm{q}}^{\mathrm{H}} \mathrm{W}_{\mathrm{RLS}, \mathrm{p})\}}^{\mathrm{H}}\right.\right.$. Here, $R_{\mathrm{e}}\{\mathbf{a}\}$ denotes the real part of $\mathbf{a}$. We assume that $\tilde{\mathrm{n}}_{\mathrm{p}, \mathrm{q}}$ is an effective noise by AWGN. Then, the sample average of $\mathrm{g}_{\mathrm{p}, \mathrm{q}}\left[\mathrm{n}_{\mathrm{p}}\right]$ is given by

$\left.<g_{\mathrm{p}, \mathrm{q}}\left[\mathrm{n}_{\mathrm{p}}\right]\right\rangle_{\mathrm{N}} \triangleq \frac{1}{\mathrm{~N}} \sum_{\mathrm{n}_{\mathrm{p}}=1}^{\left|\mathrm{T}_{\mathrm{p}}\right|} \sum_{\mathrm{p}=1}^{\mathrm{p}} \sum_{\mathrm{q}=1}^{\mathrm{Q}} \mathrm{g}_{\mathrm{p}, \mathrm{q}}\left[\mathrm{n}_{\mathrm{p}}\right]$

$$
=\left\langle\operatorname{Dg}\left(\mathrm{h}_{\mathrm{p}, \mathrm{q}} \mathrm{h}_{\mathrm{p}, \mathrm{q}}^{\mathrm{H}}\right)\right\rangle+\left\langle\tilde{\mathrm{n}}_{\mathrm{p}, \mathrm{q}}\right\rangle \mathrm{N}+\left\langle\mathrm{e}_{\mathrm{p}, \mathrm{q}}\right\rangle \mathrm{N}
$$

Where $N \triangleq|\mathcal{T} p| P Q$ represents the total number of samples for PDP estimation. $|\mathcal{T} p|$ is the number of pilot symbols at the $k p$ th subcarrier in a time slot. When is sufficiently large, the PDP can be perfectly estimated, since $\left\langle\right.$ Dg $\left.\left(h_{p, q} h_{p, q}^{H}\right)\right\rangle N \rightarrow P_{h},\left\langle\tilde{n}_{p, q}\right\rangle N \rightarrow \sigma_{n}^{2} \widetilde{w}$, and $\left\langle e_{p, q}\right\rangle N \rightarrow \mathbf{0}$. However, it is difficult for a receiver of practical MIMO-OFDM systems to obtain such a large number of samples. With an insufficient number of samples, the PDP can be approximated as $\mathrm{P}_{\mathrm{h}}\left\langle{ }^{\sim} \mathrm{Dg}\left(\mathrm{h}_{\mathrm{p}, \mathrm{q}} \mathrm{h}_{\mathrm{p}, \mathrm{q}}^{\mathrm{H}}\right)\right\rangle \mathrm{N}$

To improve the accuracy of PDP estimation with insufficient we mitigate the effective noise as follows

$\left\langle g_{\mathrm{p}, \mathrm{q}}\left[\mathrm{n}_{\mathrm{p}}\right]\right\rangle_{\mathrm{N}-} \sigma_{\mathrm{n}}^{2} \widetilde{\mathrm{w}}=\left\langle\mathrm{Dg}\left(\mathrm{h}_{\mathrm{p}, \mathrm{q}} \mathrm{h}_{\mathrm{p}, \mathrm{q}}^{\mathrm{H}}\right)\right\rangle_{+} \mathrm{Z}_{\mathrm{N}}$

where $\mathrm{Z}_{\mathrm{N}} \triangleq\left\langle\mathrm{e}_{\mathrm{p}, \mathrm{q}}\right\rangle \mathrm{N}+\left\langle\tilde{\mathrm{n}}_{\mathrm{p}, \mathrm{q}}\right\rangle \mathrm{N}-\sigma_{\mathrm{n}}^{2} \widetilde{\mathrm{w}}$ is defined as a residual noise vector, in which each entry has a zeromean. Then, the error of PDP estimation with $N$ samples can be calculated as

$\mathrm{e}_{\mathrm{N}}=\left(\left\langle\mathrm{Dg}\left(\mathrm{h}_{\mathrm{p}, \mathrm{q}} \mathrm{h}_{\mathrm{p}, \mathrm{q}}^{\mathrm{H}}\right)\right\rangle \mathrm{N}-\mathrm{P}_{\mathrm{h}}\right)+\mathrm{Z}_{\mathrm{N}}$

Since $\left[\mathrm{P}_{\mathrm{h}}\right] \mathrm{i} \geq 0$ for all $i$, the PDP can initially be estimated as

$\mathrm{P}_{\text {init }}=\frac{1}{\mathrm{~N}} \sum_{\mathrm{np}=1}^{|\mathrm{Tp}|} \sum_{\mathrm{p}=1}^{|\mathrm{P}|} \sum_{\mathrm{q}=1}^{|\mathrm{Q}|} \mathrm{S}_{\mathrm{p}, \mathrm{q}}\left[\mathrm{N}_{\mathrm{p}}\right]$

where $\mathrm{S}_{\mathrm{p}, \mathrm{q}}\left[\mathrm{N}_{\mathrm{p}}\right]$ is the sample vector of proposed PDP estimator with the $l$ th entry

$S_{\mathrm{p}, \mathrm{q}}^{\mathrm{l}}\left[\mathrm{N}_{\mathrm{p}}\right]=\left\{\begin{array}{cc}\mathrm{g}_{\mathrm{p}, \mathrm{q}}^{\mathrm{l}}\left[\mathrm{N}_{\mathrm{p}}\right]-\sigma_{\mathrm{n}}^{2} \mathrm{w}^{\sim 1} & \text { if } \mathrm{g}_{\mathrm{p}, \mathrm{q}}^{\mathrm{l}}\left[\mathrm{N}_{\mathrm{p}}\right]>\sigma_{\mathrm{n}}^{2} \mathrm{w}^{\sim 1} \\ 0 & \text { otherwise }\end{array}\right.$

where $g_{p, q}^{l}\left[N_{p}\right]=\left[g_{p, q}\left[N_{p}\right]\right] l$ and $w^{\sim 1}=[\widetilde{w}]$ To mitigate the detrimental effect of residual noise $Z_{N}$, the proposed scheme estimates the average of residual noise at the zero-taps of $\mathrm{P}_{\mathrm{h}}$. At the 1 th entry of, $\hat{\mathrm{p}}_{\text {init }}$ the zerotap can be detected as

$\mathrm{t}_{\mathrm{z}}^{1}=\left\{\begin{array}{cc}1 & \text { if } \hat{\mathrm{p}}_{\text {init }}<\beta \text { th } \\ 0 & \end{array}\right.$

otherwise

where $\beta$ th $=\frac{1}{\mathrm{Lg}} \sum_{\mathrm{Lg}=1}^{|\mathrm{Lg}-1|} \hat{p}_{\text {init }}^{\mathrm{I}}$ is defined as a threshold value for the zero-tap detection. Then, the average of residual noise at the zero-taps can be estimated as

$\hat{\mathrm{n}} \mathrm{R}_{\mathrm{avg}}=\frac{1}{\mathrm{Nz}} \sum_{\mathrm{l}=0}^{|\mathrm{Lg}-1|} \hat{\mathrm{p}}_{\text {init }}^{\mathrm{l}} \mathrm{t}_{\mathrm{z}}^{\mathrm{l}}$

where $\mathrm{N}_{\mathrm{z}}=\sum_{\mathrm{l}=0}^{\mathrm{Lg}-1} \mathrm{t}^{1} t l$ represents the total number of detected zero-taps. With the mitigation of residual noise, the lth tap of the PDP estimate, $\widehat{\mathrm{P}}_{\mathrm{h}}$, can be expressed as

$\hat{\mathrm{p}}_{\mathrm{h}}^{\mathrm{l}}=\left\{\begin{array}{cc}\hat{\mathrm{p}}_{\text {init }}^{\mathrm{l}} \mathrm{n} \mathrm{R}_{\text {avg }} & \text { if } \hat{\mathrm{p}}_{\text {init }}^{\mathrm{l}}>\widehat{\mathrm{n} R_{\text {avg }}} \\ 0 & \text { therwise }\end{array}\right.$

Then, the estimated PDP in (15) can be used to obtain the frequency-domain channel correlation in the LMMSE channel estimator.

\section{SIMULATION RESULTS}

We consider a MIMO-OFDM system. The system bandwidth is $5 \mathrm{MHz}$ with 301 subcarriers for transmitting data information and pilots at 2-GHz carrier frequency. Number of pilots are 12 . One frame consist of 14 OFDM symbols . The MIMO-OFDM system utilizes two transmit and one receive antennas $(P=2, \mathrm{Q}=1)$. The length of CP is $40(L g=40)$. One important parameter of the channel is the power delay profile which represents the average power (also called multipath intensity profile) associated with a given multipath delay. [6] For all simulations, the channel estimator is based on results.In fig 3.1 and in fig 3.2 we calculated effect of Mean square Error over variable number of samples for estimated PDP and for constant PDP respectively. The performance of proposed scheme is better than the constant power delay profile. In fig 3.3 we plotted MSE for given SNR range. The performance of two PDPs constant and estimated is observed. 


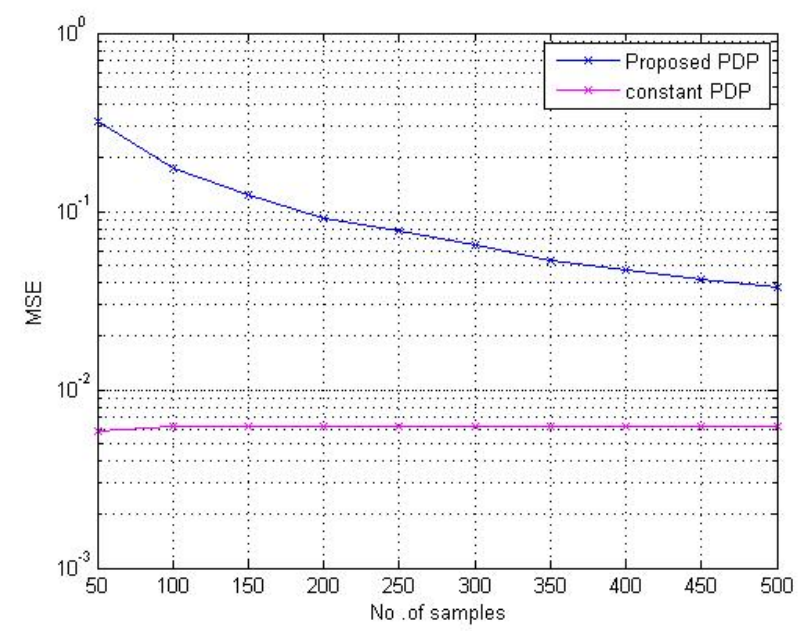

Fig :3.1 Performance of MSE with number of samples 500

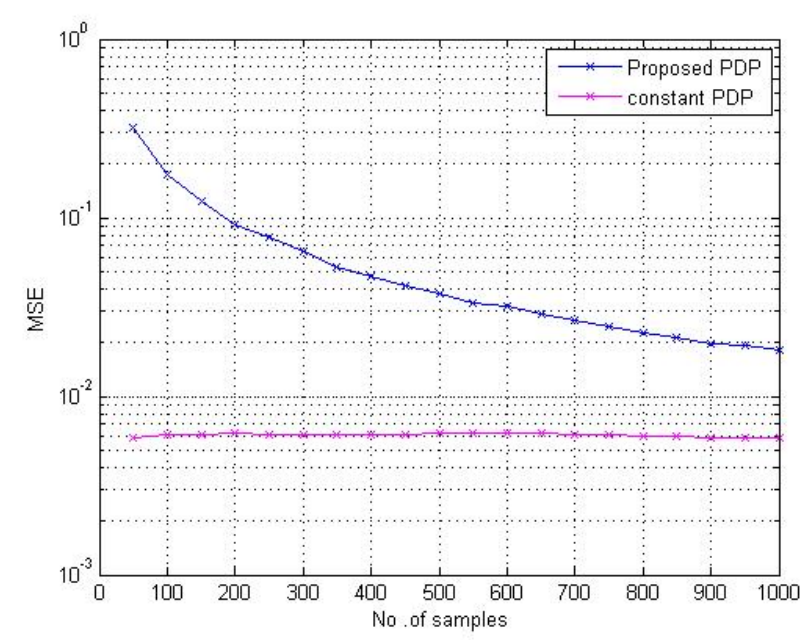

Fig :3.2 Performance of MSE with number of samples1000

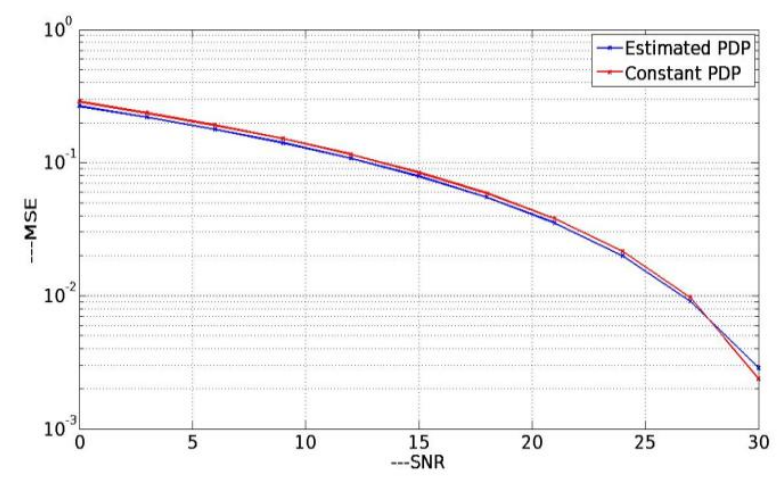

Fig :3.3 Performance of MSE with SNR

\section{CONCLUSION}

In this paper, we propose to evaluate the performance of LMMSE estimation techniques for the systems under the effect of the number of samples for estimated PDP and for constant PDP. The transmitted signals are quadrature phase-shift keying (QPSK) modulated. The cyclic prefix inserted at the beginning of each OFDM symbol is usually equal to or longer than the channel length in order to suppress ICI and ISI [5]. Simulation results show that in the case where the number of samples is increased the performance of constant PDP remains constant than the estimated PDP. MSE of the proposed scheme improves the MSE performance with an increase in the number of samples for PDP estimation. Simulation results show that the performance of LMMSE channel estimation using the proposed PDP estimate approaches that of Wiener filtering. [3]In the other case, the estimated PDP gives less SNR than constant PDP. 


\section{REFERENCES}

[1] Wang, Fei Pilot-Based Channel Estimation in OFDM System" 2011, Master of Science, University of Toledo, Electrical Engineering

[2] Sinem Coleri, Mustafa Ergen,Anuj Puri, Ahmad Bahai "A Study of Channel Estimation in OFDM Systems" 0-7803-7467-3/02/\$17.00 @2002 IEEE

[3] Young-Jin Kim and Gi-Hong Im, Senior Member, IEEE "Pilot-Symbol Assisted Power Delay Profile Estimation for MIMO-OFDM Systems" Young-Jin Kim and Gi-Hong Im, Senior Member, IEEE

[4] Keshav Kumar, Amit Grover ,"Comparison of Block Type Pilot Channel Estimation Techniques for Evaluating the performance of OFDM" International Journal of Scientific \& Engineering Research", Volume 3, Issue 11, November-2012 ISSN 2229-5518

[5] Abdelhakim Khlifil and Ridha Bouallegue "Performance Analysis of LS and LMMSE Channel Estimation Techniques for LTE Downlink Systems" International Journal of Wireless \& Mobile Networks (IJWMN) Vol. 3, No. 5, October 2011

[6] Haval Abdulrahman April 2009 , "MIMO OFDM Channel Estimation with Optimum Pilot Patterns for Cognitive Radio in Overlay Spectrum Sharing System", Thesis Number: IRCTR-A-009-09 Raffaello Tesi, Matti Hämäläinen, Jari Iinatti, "CHANNEL ESTIMATION ALGORITHMS COMPARISON FOR MULTIBAND - OFDM" The 17th Annual IEEE International Symposium on Personal, Indoor and Mobile Radio Communications (PIMRC'06)

[7] Mitalee Agrawal, Yudhishthir Raut "EFFECT OF GUARD PERIOD INSERTION IN MIMO OFDM SYSTEM" International Journal of Computer Technology and Electronics Engineering (IJCTEE) Volume 1.

[8] Kala Praveen Bagadi, Prof. Susmita Das, "MIMO-OFDM Channel Estimation using Pilot Carries", International Journal of Computer Applications (0975 - 8887) Volume 2 - No.3, May 2010

[9] Kun-Chien Hung and David W. Lin, Senior Member, IEEE, "Pilot-Based LMMSE Channel Estimation for OFDM Systems With Power-Delay Profile Approximation IEEE TRANSACTIONS ON VEHICULAR TECHNOLOGY", VOL. 59, NO. 1, JANUARY 2010 